\title{
Purpura et scorbut
}

\section{Purpura and Scurvy}

\section{Y. Touati $\cdot$ B. Oquendo $\cdot$ M. Raphael}

Reçu le 4 novembre 2017; accepté le 20 décembre 2017

(C) SFMU et Lavoisier SAS 2018

\section{Introduction}

La vitamine $\mathrm{C}$ ou acide ascorbique est une vitamine qui n'est pas synthétisée par l'être humain. Elle doit donc lui être apportée par l'alimentation. Les besoins se situent entre 110 et $120 \mathrm{mg}$ de vitamine $\mathrm{C}$ par jour pour un adulte [1]. La vitamine $\mathrm{C}$ permet de maintenir l'ion fer sous sa forme réduite Fe 2+ (propriété anti-oxydante) [2]. Ceci est nécessaire au bon fonctionnement d'une enzyme, la proline hydroxylase, qui permet la stabilité du collagène et donc du tissu conjonctif de l'organisme. L'acide ascorbique possède également un effet protecteur face aux radicaux libres [2]. Sans vitamine C, une pathologie du tissu conjonctif se développe : le scorbut [3].

Le scorbut est une pathologie connue depuis bien longtemps. Le scorbut est déjà cité sur le papyrus Ebers en 1550 avant Jésus-Christ (JC) chez les Égyptiens [3]. Le scorbut fait des ravages dans les rangs des marins dans les longues expéditions au $\mathrm{Xv}^{\mathrm{e}}$ et $\mathrm{XVI} \mathrm{I}^{\mathrm{e}}$ siècle. En 1747, James Lind, chirurgien anglais, réalisa le premier essai prospectif contrôlé de l'histoire. Le groupe mangeant deux oranges et un citron par jour se remet très rapidement du scorbut [4]. Cependant, le scorbut est une pathologie rare du passé qui peut encore se rencontrer de nos jours.

\section{Observation}

Un homme de 31 ans consulte aux urgences pour purpura des membres inférieurs. Ce patient n'a aucun antécédent, il

\author{
Y. Touati $(\bowtie) \cdot$ B. Oquendo $\cdot$ M. Raphael \\ Service d'accueil des urgences, \\ centre hospitalier universitaire Kremlin-Bicêtre, \\ 78 rue du Général Leclerc, 94270 Le Kremlin-Bicêtre, France \\ e-mail : younes.touati@outlook.com \\ Y. Touati \\ Faculté de médecine de Créteil, 8 rue du Général Raspail, \\ Créteil 94000, France \\ B. Oquendo \\ Faculté de médecine Pierre-et-Marie-Curie, \\ 91-105 boulevard de l'Hôpital, 75013 Paris, France
}

ne prend aucun traitement et ne consomme ni tabac, ni alcool ni autre substance addictogène. Depuis trois mois, il présente des épisodes de purpura des membres inférieurs dont le premier est apparu au niveau de la cuisse droite suivi d'un épanchement du genou droit. Le patient précise que, depuis sept ans, il a complètement éliminé les fruits et légumes de son alimentation en raison de troubles fonctionnels intestinaux. Il dit manger essentiellement du riz et du bœuf. Le patient présente également un problème de l'articulé dentaire l'ayant conduit à une alimentation mixée depuis trois mois.

À l'arrivée aux urgences, le patient avait une pression artérielle systolique de $130 \mathrm{mmHg}$ et une pression artérielle diastolique de $69 \mathrm{mmHg}$, une température de $36,9^{\circ} \mathrm{C}$, une fréquence cardiaque de 110 battements par minute, une saturation en oxygène périphérique en air ambiant à $100 \%$. L'examen physique était marqué par un purpura pétéchial des membres inférieurs non infiltré et centré sur le bulbe pileux. On remarquait également, comme on peut le voir sur la Figure 1, une ecchymose au niveau du pied droit et de la face interne de la cuisse droite. Enfin, l'examen des muqueuses a retrouvé une pétéchie au niveau du palais ainsi qu'une pâleur conjonctivale.

L'examen biologique était le suivant : taux d'hémoglobine $9 \mathrm{~g} / \mathrm{dL}$, un volume globulaire moyen à $110 \mathrm{fl}$, teneur corpusculaire moyenne en hémoglobine à $32 \mathrm{pg} / \mathrm{L}$, concentration corpusculaire moyenne en hémoglobine à $33 \mathrm{~g} / \mathrm{dL}$, taux de réticulocytes à $90 \mathrm{G} / \mathrm{L}$, taux de plaquettes à $135 \mathrm{G} / \mathrm{L}$. Le bilan hépatique était normal de même que le temps de Quick et le temps céphaline activateur. La prise de sang a mis en évidence une bicytopénie avec une anémie macrocytaire arégénérative et une thrombopénie. Dans ce contexte, un bilan étiologique supplémentaire a été demandé avec en particulier un dosage de vitamine B12 qui était normal avec un taux de $300 \mathrm{pg} / \mathrm{mL}$, le dosage de vitamine B9 était effondré $(1 \mu \mathrm{g} / \mathrm{L})$ pour une normale située entre 5 et $15 \mu \mathrm{g} / \mathrm{L}$, de même que le dosage vitamine $\mathrm{C}(2 \mu \mathrm{mol} / \mathrm{L})$ pour une normale supérieure à $11 \mu \mathrm{mol} / \mathrm{L}$. Le diagnostic de scorbut a été proposé.

Le patient est hospitalisé pour traitement d'épreuve. Pour ce faire, le patient a été hospitalisé en médecine interne où des sérologies VIH, VHC ont été réalisées et qui sont 


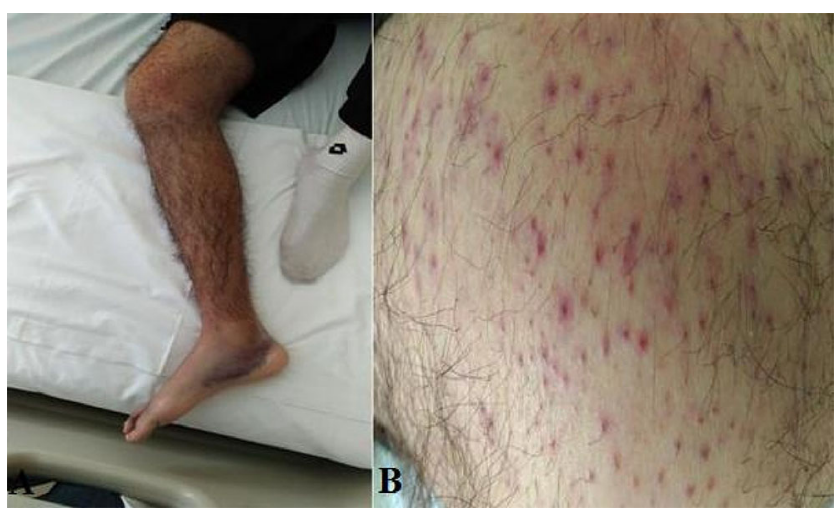

Fig. 1 A : purpura du membre inférieur droit, ecchymose de la face interne du pied droit et de la face interne de la cuisse droite ; B : purpura du membre inférieur droit centré sur le follicule pileux

revenues normales, une électrophorèse des protéines sériques a également été réalisée et était normale, de même que le dosage des anticorps anti ADN et ANCA qui était négatif. Ces explorations complémentaires réalisées en médecine interne avaient pour but d'éliminer une pathologie associée à ce scorbut pouvant expliquer le purpura, l'anémie et la thrombopénie. Aucune exploration digestive n'a été réalisée pour explorer l'anémie.

L'évolution a rapidement été favorable après introduction d'acide ascorbique à raison d'un gramme par jour en cinq jours. Avec au bout de deux jours, une diminution de la sensation d'asthénie et une disparition de la pétéchie buccale ; au bout de cinq jours, une division par trois des lésions purpuriques des membres inférieurs ; et au septième jour de l'initiation du traitement par acide ascorbique, une régression de moitié des lésions ecchymotiques.

Le diagnostic de scorbut a donc été retenu chez ce patient souffrant de troubles du comportement alimentaire avec restriction alimentaire. Il sort au bout d'une semaine d'hospitalisation avec de l'acide ascorbique $1 \mathrm{~g}$ par jour pendant 15 jours et de l'acide folique $5 \mathrm{mg}$ deux fois par jour pendant au moins un mois et une consultation de suivi à la fin du traitement. Trois jours après la fin du traitement, le patient a bénéficié d'une consultation en médecine interne qui a permis de constater que le patient ne souffrait plus des lésions purpuriques. Le patient a également repris une alimentation solide avec des fruits. Un suivi odontologique était néanmoins préconisé par l'interniste pour suivre l'évolution de sa parodontopathie ainsi que le maintien du traitement par acide folique pour suppléer la carence en vitamine B9.

\section{Discussion}

Le scorbut est une pathologie rare. Les symptômes du développement d'une carence en vitamine $\mathrm{C}$ et d'apparition du scorbut sont une asthénie majeure, des signes cutanés (pur- pura pétéchial centré sur des follicules pileux, hyperkératose périfolliculaire, dystrophie pilaire dite en queue de cochon), des signes rhumatologiques (arthralgies, myalgies), des signes hémorragiques sans trouble de l'hémostase (purpura, ecchymose, hémarthrose), des signes stomatologiques (parodontopathie) $[3,5]$. Le purpura retrouvé dans le scorbut est assez singulier puisqu'il répond aux caractéristiques d'un purpura dit plaquettaire. Il est notamment non infiltré avec lésions pétéchiales et ecchymotiques. Cependant, il est classé dans les purpuras d'origine vasculaire [6]. En effet, comme cité dans l'introduction, le scorbut est une pathologie du collagène. Du fait de l'atteinte du collagène, les vaisseaux manquent de tissu de soutien et sont par conséquent fragilisés. Le caractère périfolliculaire du purpura est également très évocateur du scorbut [3,5]. Nous rappelons dans le Tableau 1 la distinction entre un purpura plaquettaire et un purpura vasculaire.

Il semble également important de faire un rappel des causes de purpura [6] afin de mieux comprendre les explorations réalisées en médecine interne sous forme de tableau (Tableau 2).

Une anémie peut être retrouvée en cas de carence en vitamine $\mathrm{C}[3,5]$. De ce fait, une tachycardie secondaire à une anémie peut faire partie du tableau de scorbut sans que cela soit spécifique. D'autres signes biologiques peuvent être présents selon les carences associées. Une carence en vitamine B9 est souvent associée à la carence en vitamine $\mathrm{C}$ car la vitamine B9 est fournie principalement par la consommation de légumes [7]. Les symptômes de la carence en vitamine $\mathrm{C}$ s'installent progressivement. L'ascorbémie devient nulle au $41^{\mathrm{e}}$ jour, les signes cutanés apparaissent au $132^{\mathrm{e}}$ jour et les anomalies dentaires à six mois $[2,3,5]$. La consultation aux urgences intervient généralement lors de l'apparition des éléments cutanés comme le purpura.

Plusieurs études retrouvent dans la population générale un déficit en vitamine $\mathrm{C}$, défini par une valeur d'acide ascorbique inférieure à $11 \mu \mathrm{mol} / \mathrm{L}$, variant entre 5 et $14 \%: 14 \%$ des hommes et $10 \%$ des femmes de la population des ÉtatsUnis dans une étude entre 1988 et 1994 [8], $5 \%$ des femmes et $12 \%$ des hommes dans une étude chez 1108 patients de région parisienne en 1994 [9]. En 1985, une étude néerlandaise retrouve un apport de seulement $20 \mathrm{mg}$ d'acide ascorbique dans un centre de médecine physique et de

\begin{tabular}{|ll|}
\hline Tableau 1 Comparaison des purpuras plaquettaire et vasculaire \\
\hline Purpuras plaquettaires & Purpuras vasculaires \\
\hline Caractère maculeux & Caractère infiltré \\
Non infiltré & \\
Pas de prédominance déclive & Prédominance déclive \\
Atteinte muqueuse possible & Jamais d'atteinte muqueuse \\
Ecchymoses possibles & Polymorphisme lésionnel \\
\hline
\end{tabular}


Tableau 2 Étiologies les plus fréquentes de purpura

\begin{tabular}{|c|c|}
\hline Purpuras plaquettaires & Purpuras vasculaires \\
\hline $\begin{array}{l}\text { Purpura thrombopénique : cause centrale } \\
\text { ou périphérique }\end{array}$ & $\begin{array}{l}\text { Purpura infectieux : purpura fulminans, endocardite d'Osler, maladies virales } \\
\text { éruptives }\end{array}$ \\
\hline $\begin{array}{l}\text { Purpura par thrombopathie acquise surtout } \\
\text { médicamenteuse en particulier lié aux anti- } \\
\text { inflammatoires non stéroïdiens, ou lié à une } \\
\text { maladie systémique en particulier la maladie } \\
\text { de Waldenstrom, les syndromes myéloprolifératifs } \\
\text { et les myélodysplasies }\end{array}$ & $\begin{array}{l}\text { Purpura par vascularite : purpura rhumatoïde, angéite par hypersensibilité } \\
\text { aux médicaments tels que certains antibiotiques (pénicilline, cyclines, } \\
\text { sulfamides), anti-inflammatoires non stéroïdiens, maladie de Wegener, péri- } \\
\text { artérite noueuse, maladie de Churg et Strauss, purpura cryoglobulinémique, } \\
\text { purpura lié à une connectivite telle que le lupus }\end{array}$ \\
\hline \multirow{2}{*}{$\begin{array}{l}\text { Purpura par thrombopathie constitutionnelle } \\
\text { en particulier la maladie de Willebrand }\end{array}$} & Purpura lié à une amylose \\
\hline & $\begin{array}{l}\text { Purpura par anomalies constitutionnelles du vaisseau : maladie d'Ehlers- } \\
\text { Danlos, fragilité capillaire constitutionnelle } \\
\text { Purpura du scorbut lié à une anomalie du collagène }\end{array}$ \\
\hline
\end{tabular}

réadaptation néerlandais. Seuls ceux qui recevaient des fruits et des légumes de l'extérieur ne sont pas carencés [10]. Enfin, certaines populations sont plus touchées que d'autres. Dans une étude réalisée à Paris en 2002, sur 87 sans domiciles fixes dont $90 \%$ déclaraient une intoxication alcoolique ou tabagique, $72 \%$ de ces patients présentaient une forte carence en vitamine $\mathrm{C}$ (taux d'acide ascorbique indétectable) [11].

On estime qu'en France, le risque de scorbut atteindrait 15 à $25 \%$ des personnes de plus de 65 ans $[9,12]$ ce qui en fait une population importante à surveiller. Le deuxième point est celui de l'isolement social. En effet, des cas de personnes atteintes de scorbut vivant seules, comme les célibataires, les veufs(ves) ou en institution ont été mis en évidence [12]. Le chômage et la retraite sont associés à un taux plus faible d'acide ascorbique dans le sang, favorisé par l'isolement [12]. Les autres facteurs de risque sont le tabagisme, l'alcoolisme, les troubles alimentaires liés à des cancers, les pathologies de malabsorption, la grossesse, les troubles psychiatriques dont notamment l'anorexie mentale, les régimes stricts sans apport de fruits ou légumes [12].

Le traitement curatif est simple, peu onéreux et repose sur la supplémentation orale ou intraveineuse, à raison de $1 \mathrm{~g} / \mathrm{j}$ d'acide ascorbique pendant 15 jours. Les apports en vitamine $\mathrm{C}$ pour $100 \mathrm{mg}$ d'aliments sont [13] : $40 \mathrm{mg}$ pour une orange, $55 \mathrm{mg}$ pour un citron, $140 \mathrm{mg}$ pour un poivron, $190 \mathrm{mg}$ pour du persil. Le jus d'orange le moins cher contient au minimum $300 \mathrm{mg}$ de vitamine C par litre [14]. L'efficacité du traitement est rapide et spectaculaire en cinq à huit jours, confirmant le diagnostic [3].

En conclusion de ce cas, un scorbut doit être évoqué devant des signes évocateurs, a fortiori chez les patients à risque car la carence en vitamine $\mathrm{C}$ est loin d'être réservée aux pays pauvres.
Liens d'intérêts : Les auteurs déclarent ne pas avoir de liens d'intérêts.

\section{Références}

1. Levine M, Rumsey SC, Daruwala R, et al (1999) Criteria and recommendations for vitamin C intake. JAMA 281:1415-23

2. Padayatty SJ, Levine M (2001) New insights into the physiology and pharmacology of vitamin C. CMAJ 164:353-5

3. Fain O (2004) Carences en vitamine C. Rev Med Int 25:872-80

4. Bartholomew M (2002) James Lind's treatise of the scurvy (1753). Postgrad Med J 78:695-6

5. Hodges RE, Hood J, Canham JE, et al (1971) Clinical manifestations of ascorbic acid deficiency in man. Am J Clin Nutr 24:432-43

6. Ifrah N (2014) Purpura. In: Société française d'hématologie, Hématologie. Elsevier Masson Ed, Paris, pp 193-8

7. Sauvant P, Azaiis-Braesco V (2001) Vitamines liposolubles. In: Basdevant A, Laville M, Lerebours E, Traité de nutrition clinique de l'adulte. Médecine-Sciences Flammarion Ed, Paris, pp 183-93

8. Hampl JS, Taylor CA, Johnston CS (2004) Vitamin C deficiency and depletion in the United States: The Third National Health and Nutrition Examination Survey, 1988 to 1994. Am J Public Health 94:870-5

9. Hercberg S, Preziosi P, Galan P, et al (1994) Vitamin status of a healthy French population: dietary intakes and biochemical markers. Int J Vitam Nutr Res 64:220-32

10. Berkhout AM, Hoen J (1985) Vitamin C deficiency in patients in a nursing home. Tijdschr Gerontol Geriatr 16:3-7

11. Malmauret L, Leblanc J, Cuvelier I, Verger P (2002) Dietary intakes and vitamin status of a sample of homeless people in Paris. Eur J Clin Nutr 56:313-20

12. Fain O, Pariés J, Jacquart B, et al (2003) Hypovitaminosis C in hospitalized patients. Eur J Intern Med 14:419-25

13. Agence nationale de sécurité sanitaire de l'alimentation, de l'environnement et du travail (ANSES) (2017) Ciqual Table de composition nutritionnelle des aliments. https://ciqual.anses.fr/ (Dernier accès le 4 novembre 2017)

14. Garnier C (2011) Jus de fruits. https://www.quechoisir.org/guided-achat-jus-de-fruits-n9169/ (Dernier accès le 4 novembre 2017) 